EDITORIAL

\title{
The extents of mitral leaflet opening and closure are determined by left ventricular systolic function
}

\section{S Kaul}

Heart 2004;90:126-128. doi: 10.1136/hrt.2003.014373

The extent of opening of the mitral leaflets in diastole and their degree of closure during systole are mediated either indirectly or directly through the force of left ventricular systole. Therefore measures of incomplete opening or incomplete closure both reflect left ventricular systolic function
Correspondence to:

Saniiv Kaul, MD,

Cardiovascular Division, Box 800158, Medical Center, University of Virginia, Charlottesville VA 22908-0158, USA; sk@virginia.edu $\mathrm{n}$ order to appreciate the spatiotemporal relation between the mitral leaflets and mitral annular plane during the cardiac cycle, one has to begin with the "neutral" position of the mitral leaflets in relation to the mitral annular plane. This can be defined as the position of the mitral leaflets when there is no pressure difference between the left atrium and left ventricle. If the annulus were a Euclidean plane, the neutral position of the leaflets would be in the plane of the annulus (fig 1A). Because the mitral annulus is saddle shaped ${ }^{1}$ the neutral position of the mitral leaflets is not in the Euclidean plane but is caudal to it (fig 1B). This position results from the tension on the leaflets by the saddle shaped annulus which affords them a spring-like quality so that a greater force is required to move them cephalad towards the mitral annular plane than to move them caudally further into the left ventricular (LV) cavity (fig 1B).

\section{NORMAL LV FUNCTION}

Let us begin at this neutral position at the time of left atrial (LA)-LV pressure crossover when the mitral valve opens (Co in fig 2, with the corresponding two dimensional echocardiographic orientation in fig 3). The mitral leaflets are pulled into the LV due to the rapidly changing LA-LV pressure gradient that occurs early in diastole ( $\mathrm{O}$ in fig 2 , with the corresponding two dimensional echocardiographic orientation in fig 3). For any given LA pressure and LV afterload, the rate at which the diastolic LA-LV gradient increases depends on the rate of LV elastic recoil, which in turn is determined by LV systolic function: the worse the LV systolic function, the slower the rate of LV elastic recoil $^{2}{ }^{3}$; the slower the rate of $\mathrm{LV}$ elastic recoil, the slower the rate of rise in diastolic LA-LV pressure gradient. When the rate of rise of the diastolic LA-LV pressure gradient is rapid, the degree of mitral leaflet opening is greater and the degree of E point septal separation (EPSS) is smaller. Conversely, a slow rate of rise in this gradient causes a smaller degree of mitral leaflet opening and a greater degree of EPSS. It is not the absolute change in pressure that matters but the rate of rise of pressure.

After the leaflets have attained their maximal opening they start drifting cephalad, even before the occurrence of peak forward mitral flow, ${ }^{4}$ and the leaflet tips coapt in the neutral position caudal to the annulus. The leaflets assume their neutral position because the LA-LV gradient is zero or minimal during atrial diastasis ( $\mathrm{d}$ in fig 2 , with the corresponding two dimensional echocardiographic orientation in fig 3). The left atrium then contracts at the end of atrial diastasis, increasing the LA-LV pressure gradient and once again causing the mitral leaflets to move caudally and towards the LV walls. The degree of mitral valve opening is less during atrial systole than early diastole $\left(\mathrm{O}^{\prime}\right.$ in fig 2 , with the corresponding two dimensional echocardiographic orientation in fig 3 ). When the diastolic LA-LV pressure gradient approaches zero towards the end of atrial systole, the leaflets again drift towards their neutral position $\left(\mathrm{Co}^{\prime}\right.$ in fig 2, with the corresponding two dimensional echocardiographic orientation in fig 3). The leaflets coapt at this position but do not "close".

Once LV systole starts and the systolic LV-LA pressure gradient increases above a critical level, it overcomes the spring-like tension in the mitral leaflets and pushes them towards the mitral annular plane, thus closing them $\left(\mathrm{Cl}^{\prime}\right.$ in fig 2 , with the corresponding two dimensional echocardiographic orientation in fig 3). Whereas the onset of LV systole is not required for coaptation of the mitral leaflets at the end of atrial systole, it is LV systole that causes the cephalad motion of the mitral leaflets towards the mitral annular plane, resulting in complete closure of the leaflets. Under normal conditions, the distance between mitral leaflet coaptation point and mitral annular plane in systole is approximately $0.25 \mathrm{~cm}$.

\section{SYSTOLIC DYSFUNCTION}

In the presence of LV systolic dysfunction, the rate of rise of the systolic LV-LA pressure gradient determines the degree to which the mitral leaflets close in systole. The slower this rate of rise of pressure, the greater the distance between the mitral leaflet coaptation point and the mitral annular plane in systole (incomplete mitral leaflet closure (IMLC)). Again, it is the

Abbreviations: EPSS, E point septal separation; IMLC, incomplete mitral leaflet closure; LA, left atrium; LV, left ventricle 


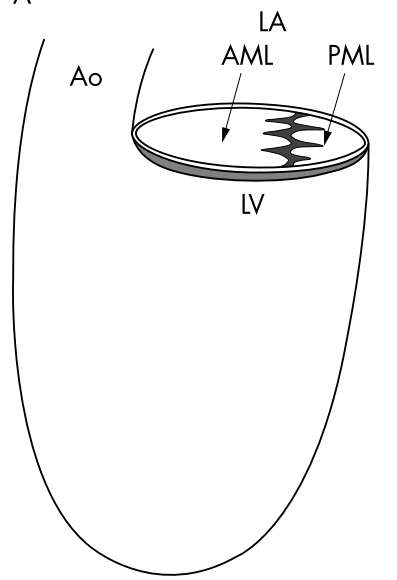

B

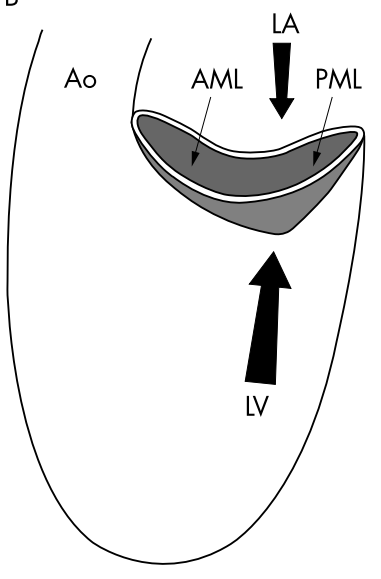

Figure 1 Effect of the shape of the mitral annulus on the neutral position of the mitral leaflets. If the annulus is in the Euclidean plane, the leaflet coaptation plane will remain within that same plane (A). When the annulus is saddle shaped, the leaflet coaptation plane will move caudally (B). A greater force is required to move the leaflets cephalad towards the mitral annular plane (large arrow) than to move them caudally further into the left ventricular cavity (small arrow). AML, anterior mitral leaflet; Ao, aorta; LA, left atrium; LV, left ventricle; PML, posterior mitral leaflet. Reproduced from Dent et al ${ }^{\prime}$ with permission of the American Physiological Society.

rate of rise of pressure rather than reaching an absolute pressure that is important.

During isovolumic relaxation and decline in LV pressure, when LV-LA pressure gradient reaches a level where the force against the spring is not enough to keep it at the annular plane, the spring recoils using the energy stored in it during peak systole, and the leaflet coaptation point moves back to the neutral position prior to LA-LV pressure crossover, before mitral flow starts again and the cardiac cycle is repeated.

What is the difference between mitral leaflet coaptation and closure in systole? For leaflets to close fully, their rough edges must approximate each other and the sphincteric action of the mitral annulus must seal these edges together, otherwise mitral regurgitation can occur. It has been shown that the sphincteric action of the mitral annulus is reduced in several conditions, including IMLC and mitral annular calcification. In these situations the mitral regurgitation is central and its severity is roughly proportional to the degree of IMLC or mitral annular calcification. Placing an annular ring during surgery allows better approximation and sealing of the mitral leaflets in patients with global (and not regional) ischaemic LV systolic dysfunction. Revascularising viable myocardium in these patients assures recovery in LV function and reduction in the degree of IMLC as well as mitral regurgitation.

There is a good correlation between the degree of mitral leaflet opening and the magnitude of EPSS, indicating that one can be used as a surrogate of the other. LV elastic recoil determines the magnitude of EPSS and LV elastic recoil itself is determined by LV systolic function. This finding is in keeping with results of previous studies that have shown a good inverse correlation between the degree of EPSS and LV ejection fraction..$^{5-7}$ Not surprisingly, therefore, in the absence of aortic regurgitation, where the regurgitant jet can limit anterior mitral leaflet excursion, ${ }^{8}$ the degree of EPSS has been correlated with outcome in patients with various forms of cardiovascular disease. ${ }^{9}$ Interestingly, although EPSS is a diastolic measurement, it reflects systolic LV function, indicating that most of the diastolic behaviour of the left ventricle is influenced by its systolic behaviour.

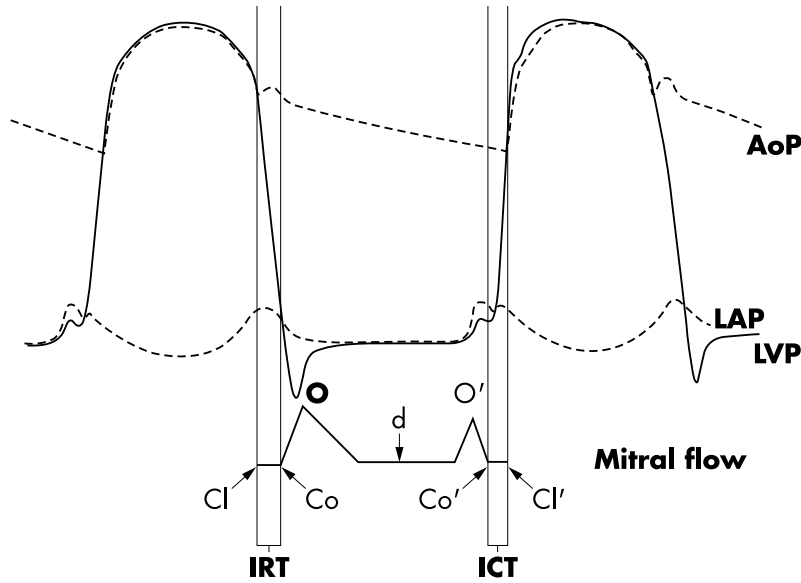

Figure 2 Haemodynamics and mitral valve flow during the cardiac cycle. AoP, aortic pressure; ICT, isometric contraction time; IRT, isovolumic relaxation time; LAP, left atrial pressure; LVP, left ventricular pressure; $\mathrm{Cl}, \mathrm{Co}, \mathrm{O}, \mathrm{d}, \mathrm{O}^{\prime}, \mathrm{Co}^{\prime}$ and $\mathrm{Cl}^{\prime}$ are defined in the text.

\section{ROLE OF PAPILLARY MUSCLES}

The degree of IMLC has also been measured in the clinical setting. ${ }^{10-13}$ It was initially felt that the degree of IMLC noted in ischemic heart disease occurs because of dyskinesia of the LV free wall in patients with myocardial infarction, which pulls the papillary muscle outward resulting in a caudal coaptation of the mitral leaflets in systole. ${ }^{14}$ It has since been demonstrated that this phenomenon occurs in nonischaemic LV dysfunction as well. ${ }^{11}{ }^{13}{ }^{15}$ It has been shown that when papillary muscle function is kept normal (by perfusing it selectively with blood), the degree of IMLC correlates with the degree of LV systolic dysfunction. ${ }^{15}$

The main role of the papillary muscles and the chordae tendinae is prevention of mitral valve prolapse in systole. Once the systolic LV-LA pressure gradient exceeds the spring-like tension of the leaflets and pushes them towards the mitral annular plane, movement of the leaflets cephalad to this plane is prevented by these structures. It may not even be necessary for the papillary muscles to shorten during systole in order to prevent prolapse of the mitral leaflets. It has been reported that the distance between the mitral leaflet tips and the LV apex remains constant throughout the cardiac cycle, suggesting that the papillary muscles contract isometrically. ${ }^{16}$ It has also been shown that while ischaemia of one or both papillary muscles results in their lack of thickening, it does not result in prolapse. ${ }^{15}$ Ischaemia of the papillary muscles does not result in greater degree of IMLC either. The degree of IMLC does not increase during lack of thickening of the papillary muscles and the immediately adjacent LV wall. ${ }^{15} 1718$

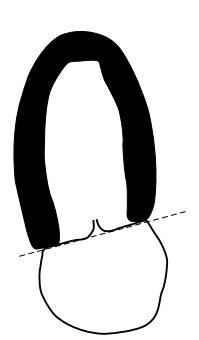

$\mathrm{Cl}, \mathrm{Cl}^{\prime}$

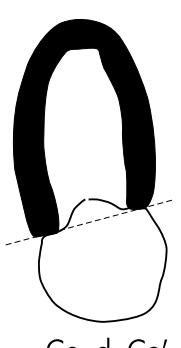

Co, d, Co'

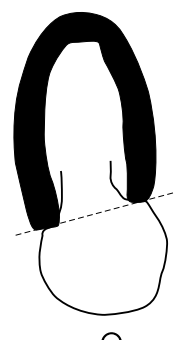

$\mathrm{O}$

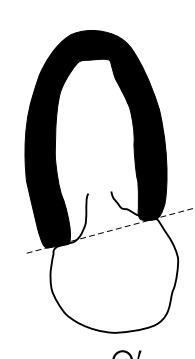

$\mathrm{O}^{\prime}$
Figure 3 Positions of the mitral leaflets in relation to the annular plane at different times of the cardiac cycle. $\mathrm{Cl}, \mathrm{Co}, \mathrm{O}, \mathrm{d}, \mathrm{O}^{\prime}, \mathrm{Co}^{\prime}$ and $\mathrm{Cl}^{\prime}$ are defined in the text. 
The diastolic LA-LV pressure gradient influences the magnitude of forward mitral valve flow in the same manner in which it influences the degree of mitral leaflet opening (fig 2), but the magnitude of forward mitral flow is not responsible for mitral leaflet opening. When forward mitral valve flow is increased during severe LV dysfunction by transiently increasing LA pressure, the degree of IMLC remains unchanged. Adequate mitral valve opening is seen despite no flow across the mitral valve when the pulmonary veins are suddenly occluded in a beating canine heart without changing LV function. Therefore, the reduction of IMLC during dobutamine administration noted by Karagiannis and colleagues ${ }^{13}$ in a recent issue of Heart is due to the direct effect of the drug on LV systolic function, rather than its secondary effect on cardiac output.

A word of caution on measuring LV systolic function using LV ejection fraction. As a measure of LV systolic function, this parameter is as misleading as it is easy to measure. It is highly load dependent. LV end systolic dimensions, LV peak systolic wall stress, and newer tissue Doppler derived parameters more accurately reflect LV systolic function and should be measured more frequently in echocardiographic laboratories, particularly when LV systolic function is a major determinant of an issue under study.

\section{CONCLUSION}

Both the extents of opening of the mitral leaflets in diastole and their degree of closure during systole are mediated either indirectly (opening) or directly (closure) through the force of LV systole. Therefore measures of incomplete opening (increased EPSS) or incomplete closure (IMLC) both reflect LV systolic function. IMLC also results in mitral regurgitation with a central jet, the degree of which is roughly proportional to the degree of IMLC. In ischaemic mitral regurgitation associated with reduced global LV systolic function but viable myocardium, and IMLC with intact mitral leaflets, a mitral ring in conjunction with revascularisation is all that is necessary to assure subsequent reduction in the degree of mitral regurgitation in association with improvement in LV systolic function.

\section{ACKNOWLEDGEMENTS}

The author thanks Kevin Wei, MD for executing the artwork.

\section{REFERENCES}

1 Levine RA, Triulzi MO, Harrigan $P$, et al. The relationship of mitral annular shape to the diagnosis of mitral valve prolapse. Circulation 1987;75:756-67.

2 Dent JM, Spotnitz WD, Nolan SP, et al. Mechanism of mitral leaflet excursion. Am J Physiol 1995;269:H2100-8.

3 Courtois M, Mechem CJ, Barzilai B, et al. Factors related to end-systolic volume are important determinants of peak early diastolic transmitral flow velocity. Circulation 1992;85:1132-8.

4 Laniado $\mathrm{S}$, Yellin $\mathrm{E}$, Kotler $\mathrm{M}$, et al. A study of the dynamic relations between the mitral valve echogram and phasic mitral flow. Circulation 1975;51:104-13

5 Ahmadpour H, Shah AA, Allen JW, et al. Mitral E point septal separation: a reliable index of left ventricular performance in coronary artery disease. Am Heart J 1983;106:21-8

6 Child JS, Krivokapich J, Perloff JK. Effect of left ventricular size on mitral E point to ventricular septal separation in assessment of cardiac performance. Am Heart J 1981;101:797-805.

7 Massie BM, Schiller NB, Ratshin RA, et al. Mitral-septal separation: new echocardiographic index of left ventricular function. Am J Cardiol 1977;39:1008-16

8 Nolan SP, Fisher RD, Dixon SH Jr, et al. Alterations in left atrial transport and mitral valve blood flow resulting from aortic regurgitation. Am Heart $J$ 1970;79:668-75.

9 Engle SJ, DiSessa TG, Perloff JK, et al. Mitral valve E point to ventricular septal separation in infants and children. Am J Cardiol 1983;52:1084-7.

10 Godley RW, Wann LS, Rogers EW, et al. Incomplete mitral leaflet closure in patients with papillary muscle dysfunction. Circulation 1981;63:565-71.

11 Kaul S, Pearlman JD, Touchstone DA, et al. Prevalence and mechanisms of mitral regurgitation in the absence of intrinsic abnormalities of the mitral leaflets. Am Heart J 1989;118:963-72.

12 Kinney EL, Frangi MJ. Value of two-dimensional echocardiographic detection of incomplete mitral leaflet closure. Am Heart J 1985;109:87-90.

13 Karagiannis SE, Karatasakis GT, Koutsogiannis N, et al. Increased distance between mitral leaflet coaptation point and mitral annular plane: significance and correlation in patients with heart failure. Heart 2003;89:1174-8.

14 Tsakiris AG, Von Bernuth G, Rastelli GC, et al. Size and motion of the mitral valve annulus in anesthetized dog hearts. J Appl Physiol 1971;30:611-8.

15 Kaul S, Spotnitz WD, Glasheen WP, et al. Mechanism of ischemic mitral regurgitation: an experimental evaluation. Circulation 1991;84:2167-80.

16 Karas S, Elkins RC. Mechanism of function of the mitral valve leaflets, chordae tendineae, and left ventricular papillary muscles in dogs. Circ Res 1970;26:689-96.

17 Mittal AK, Langston M, Cohn KE, et al. Combined papillary muscle and left ventricular wall dysfunction as a cause of mitral regurgitation: an experimental study. Circulation 1971:44:174-80.

18 Tsakiris AG, Rastelli GC, Amorim DD, et al. Effect of experimental papillary muscle damage on mitral valve closure in intact anesthetized dogs. Mayo Clin Proc 1970;45:275-85.

\section{ELECTRONIC PAGES}

\section{Heart Online case reports: www.heartjnl.com}

7

he follow electronic only articles are published in conjunction with this issue of Heart.

\section{Tachycardiomyopathy: a diagnosis not to be missed} N L Walker, S M Cobbe, D H Birnie

The prognosis of dilated cardiomyopathy is generally poor. In the vast majority of cases the cause of the ventricular dysfunction is irreversible but occasionally potentially curable causes are identified. Tachycardiomyopathy is a rare and potentially treatable cause of heart failure. A patient with a particularly severe case who had an excellent outcome is presented.
(Heart 2004;90:e7) www.heartjnl.com/cgi/content/full/90/ $2 / \mathrm{e} 7$

Computed tomographic virtual cardioscopy in a case of left atrial myxoma

$\mathrm{H}-\mathrm{W}$ Chen, S-J Chen, I-S Chiu

Computed tomographic virtual cardioscopy was used to provide clear and precise visualisation of a myxoma with a stalk arising from the interatrial septum. This technique permits the safe, reliable, and non-invasive diagnosis of intracardiac lesions. This case is presented to assist the cardiovascular surgeon in preoperative planning or in developing a simulation of robotic cardiac surgery. $2 / \mathrm{e} 8$ 